\title{
Erratum: Dark photon from light scalar boson decays at FASER
}

\section{T. Araki, ${ }^{a}$ K. Asai, ${ }^{b}$ H. Otono, ${ }^{c}$ T. Shimomura ${ }^{d}$ and Y. Takubo ${ }^{e}$}

${ }^{a}$ Faculty of Dentistry, Ohu University, 31-1 Sankakudo, Tomita-machi, Koriyama, Fukushima 963-8611, Japan

${ }^{b}$ Department of Physics, University of Tokyo,

7-3-1 Hongo, Bunkyo-ku, Tokyo 133-0033, Japan

${ }^{c}$ Research Center for Advanced Particle Physics, Kyushu University, Fukuoka 819-0395, Japan

${ }^{d}$ Faculty of Education, University of Miyazaki, 1-1 Gakuen-Kibanadai-Nishi, Miyazaki 889-2192, Japan

${ }^{e}$ Institute of Particle and Nuclear Study, KEK, Oho 1-1, Tsukuba, Ibaraki 305-0801, Japan

E-mail: t-araki@den.ohu-u.ac.jp, asai@hep-th.phys.s.u-tokyo.ac.jp, otono@phys.kyushu-u.ac.jp, shimomura@cc.miyazaki-u.ac.jp, yosuke.takubo@kek.jp

ERRATUM TO: JHEP03(2021)072

ArXiv EPrint: 2008.12765 

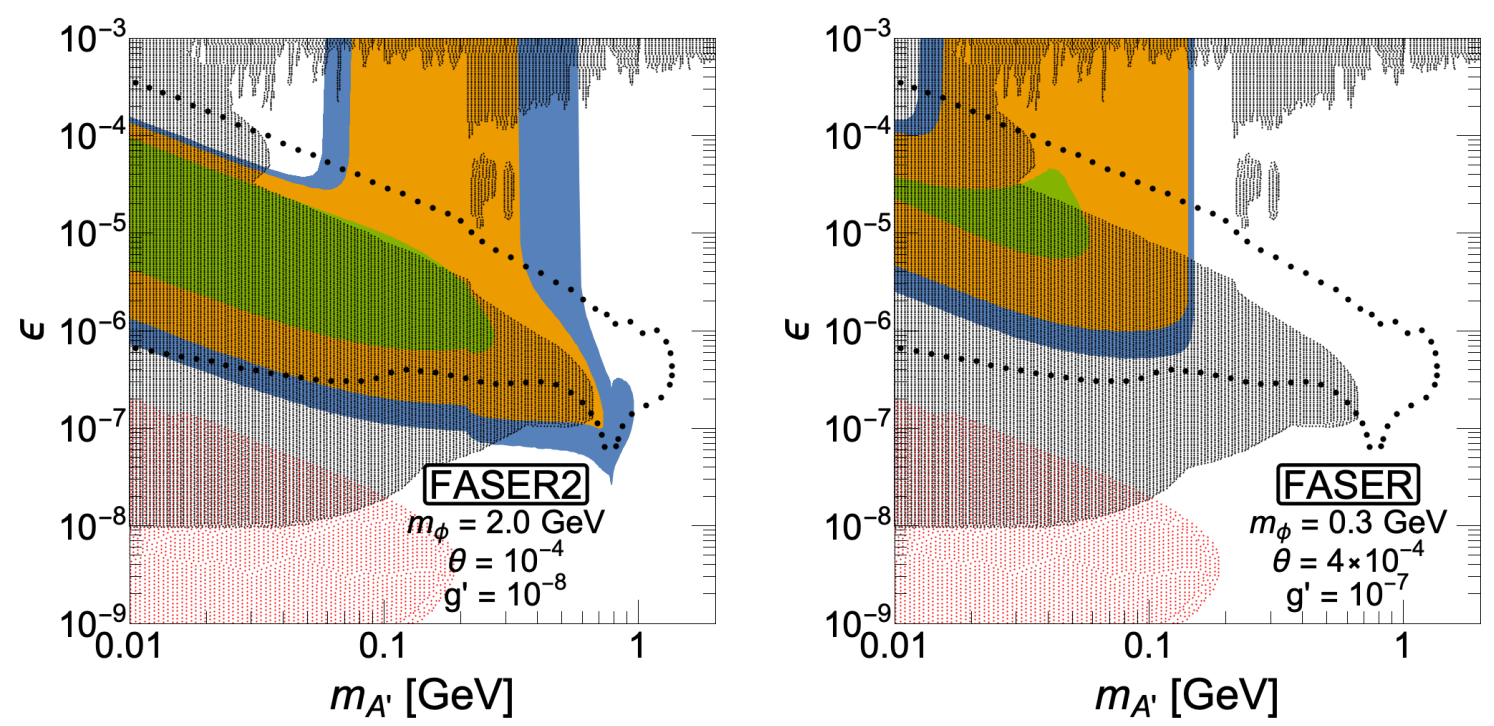

Figure 1. Expected number of the signal events of the dark photon after correcting the $\phi$ decay width.

We have found a mistake in calculations of the expected number of the signal events shown in figure 2, 4 and 5 of our paper. The decays of the scalar boson $\phi$ into hadrons were not appropriately included in its branching ratio in eq. (4.7). The correction affects the results only for the cases of small $g^{\prime}$, namely the right panels in figures 2,4 and 5 . For the cases of FASER2, the number of the signal events is reduced by including the correction, in particular in the large $m_{A^{\prime}}$ regions where the enhancement due to $m_{\phi} \gg m_{A^{\prime}}$ in eq. $(2.2)$ is weak. In contrast, for the case of FASER, we have found that the signal regions are shrunk by the correction even in small $m_{A^{\prime}}$ regions. The results including the correction are shown in figure 1 in this erratum for figure 2 (left panel) and figure 4 (right panel) of the original paper, while the changes due to the correction are negligible for figure 5 . The changes in other results with $g^{\prime} \geq 10^{-6}$ are negligible because the decay width into dark photons is enough larger than that into hadrons.

Open Access. This article is distributed under the terms of the Creative Commons Attribution License (CC-BY 4.0), which permits any use, distribution and reproduction in any medium, provided the original author(s) and source are credited. 